\title{
Perfil de resistencia bacteriana en hospitales y clínicas en el departamento del Cesar (Colombia)
}

\author{
Bacterial resistance profile and clinical hospital \\ department of Cesar (Colombia)
}

María C. Yaneth-Giovanetti MSc ${ }^{1}$,
Gloria I. Morales-Parra MSc ${ }^{2}$,
Cielo Armenta-Quintero Bact ${ }^{3}$

Introducción: la resistencia a los antimicrobianos es un problema de salud pública cuyo tratamiento puede ser difícil y costoso. Objetivo: analizar el fenotipo de resistencia antimicrobiana en bacterias aisladas en hospitales y clínicas del departamento del Cesar (Colombia) durante 2014. Materiales y métodos: se realizó un estudio descriptivo en cinco laboratorios clínicos del departamento. Se utilizó el software Whonet 5.6 para el análisis de datos y se reportaron los perfiles de resistencia. Resultados: las principales bacterias Gram negativas aisladas en la Unidad de Cuidados Intensivos fueron KlebsieIla pneumoniae (18,8\%), Pseudomonas aeruginosa $(15,0 \%)$ y Escherichia coli $(13,8 \%)$. En los demás servicios predominaron Escherichia coli $(36,4 \%)$ y Staphylococcus aureus (15,5\%). En la Unidad de Cuidados Intensivos la mayor resistencia fue a la ampicilina-sulbactam y las cefalosporinas de tercera y cuarta generación en los aislados de Klebsiella pneumoniae $(46,2 \%, 28,3 \%$ y $29,1 \%$, respectivamente) y de Escherichia coli (21,8\%, 21,8\% y 23,0\%, respectivamente). En los aislados de Acinetobacter la resistencia fue mayor que en los de Pseudomonas aeruginosa, con predominio a la ceftriaxona y la cefepima $(51,1 \%)$ en los aislados de la Unidad de Cuidados Intensivos. En los aislamientos de Staphylococcus aureus y Staphylococcus epidermidis se observó resistencia a la oxacilina en el 61,0\% y 81,8\% de los provenientes de la Unidad de Cuidados Intensivos, y en el $48,7 \%$ y el $89,7 \%$ de las demás unidades, respectivamente. Conclusiones: se observaron altas frecuencias de resistencia antibiótica por lo que reforzar la vigilancia epidemiológica a nivel local permitirá disminuir la resistencia bacteriana en los ambientes intrahospitalarios.

Palabras clave: farmacorresistencia bacteriana, vigilancia epidemiológica, bacterias Grampositivas, Bacterias Gramnegativas.

Yaneth-Giovanetti MC, Morales-Parra GI, Armenta-Quintero C. Perfil de resistencia bacteriana en hospitales y clínicas en el departamento del Cesar (Colombia). Medicina \& Laboratorio 2017; 23: 387-398.

${ }^{1}$ Bacterióloga, MSc en Ciencias Biológicas (Microbiología). Docente Universidad de Santander (UDES), Facultad de Ciencias de la Salud, Grupo de Investigación Cienciaudes. Valledupar, Colombia. Correo electrónico: maceya@gmail.com

${ }^{2}$ Bacterióloga y Laboratorista Clínico, MSc en Microbiología Molecular. Docente Universidad de Santander (UDES), Facultad de Ciencias de la Salud, Grupo de Investigación Cienciaudes. Valledupar, Colombia.

${ }^{3}$ Bacterióloga, Especialista en Epidemiologia. Profesional especializado del Laboratorio de Salud Pública del Departamento del Cesar. Valledupar, Colombia.

Conflicto de intereses: los autores declaran que no tienen conflicto de intereses Medicina \& Laboratorio 2017; 23: 387-398

Módulo 19 (Investigación), número 59. Editora Médica Colombiana S. A. 2017 Recibido el 26 de julio de 2017; aceptado el 30 de agosto de 2017 
a Organización Mundial de la Salud (OMS), en el año 2001, declaró la resistencia a los antimicrobianos como un problema de salud pública y anunció la estrategia mundial para la contención de la resistencia a los antimicrobianos [1]. Esta problemática hace que el tratamiento sea difícil, costoso e incluso imposible en algunos casos. El impacto en los pacientes vulnerables es más evidente, lo que resulta en una enfermedad prolongada y aumento de la mortalidad [2].

La magnitud del problema de la resistencia a los antimicrobianos es a nivel mundial, debido a su impacto en la salud humana, los costos para la salud y el problema que representa en la sociedad en general. Algunas estimaciones de los efectos económicos de la resistencia a los antimicrobianos han mostrado resultados inquietantes; por ejemplo, en Estados Unidos representan un costo anual para el sistema de salud entre USD $\$ 21.000 .000 .000$ y USD $\$ 34.000 .000 .000$, donde más de USD $\$ 8.000 .000$ se generan por día adicional de permanencia en el hospital de cada paciente resistente [2].

El ámbito hospitalario es un componente crítico de la problemática de la resistencia a los antimicrobianos, donde la combinación de factores como la presencia de pacientes altamente susceptibles, la implementación cada vez mayor de dispositivos médicos invasivos, el uso intensivo y prolongado de antibióticos y la presencia de infecciones cruzadas pueden llevar a infecciones asociadas a la atención en salud por microorganismos resistentes que desencadenan la emergencia y diseminación de este fenómeno [3].

En Colombia se propuso poner en práctica, a nivel nacional, la vigilancia de la resistencia bacteriana y el consumo de antibióticos; este último a manera de una primera aproximación al conocimiento de su uso, que permita abordar el problema desde otras perspectivas relacionadas con el aumento de la resistencia bacteriana, la elevada carga de infecciones y el uso inapropiado de antibióticos en los hospitales [4]. Es así como la vigilancia de la resistencia a los antimicrobianos es indispensable para obtener información sobre la magnitud y las tendencias del problema, y para realizar el seguimiento del efecto de las intervenciones.

En el departamento del Cesar (Colombia), hasta la fecha, no se contaba con datos generales sobre el perfil de resistencia bacteriana, ya que solo existían los datos aislados de cada institución hospitalaria local. Por lo tanto, la obtención de un consolidado de la información sobre los fenotipos de resistencia en este departamento es necesaria para determinar su dimensión y frecuencia, e implementar la vigilancia en resistencia bacteriana que, a pesar de que se encuentra reglamentada desde el año 2012 por el Instituto Nacional de Salud en el SIVIGILA, no se realiza. El objetivo de esta investigación fue analizar el comportamiento de la resistencia antimicrobiana en patógenos bacterianos aislados en hospitales y clínicas en el departamento del Cesar durante el año 2014.

\section{Materiales y métodos}

Se realizó un estudio descriptivo y retrospectivo en cinco laboratorios clínicos de los hospitales y clínicas adscritos a la Red de Laboratorios del departamento del Cesar, que realizaron cultivos bacterianos y pruebas de susceptibilidad en el año 2014. La información se obtuvo del consolidado mensual y anual de los resultados de los cultivos y an- 
tibiogramas de las muestras clínicas de los pacientes hospitalizados que fueron proporcionados por las instituciones participantes.

La base de datos obtenida fue transferida al programa WHONET versión 5.6 utilizando la aplicación BacLink (WHO Collaborating Centre for Surveillance of Antimicrobial Resistance, Massachusetts, Estados Unidos), donde se filtraron y unificaron los datos de interés provenientes de los diferentes laboratorios. El análisis de la información se realizó en WHONET y se resumió en un formato de extracción de datos de Microsoft Excel, en el que se establecieron las proporciones de los microorganismos más prevalentes por localización (instalación) dentro del área hospitalaria, bien sea la Unidad de Cuidados Intensivos ( $\mathrm{UCI}$ ), donde ocurren la mayoría de infecciones asociadas a la atención en salud [5,6], o los servicios diferentes a esta (hospitalización, excepto emergencias), discriminando por año calendario.

A partir de la información consignada se determinó el número de aislamientos de Escherichia coli, Klebsiella pneumoniae, Pseudomonas aeruginosa, Acinetobacter baumannii, Staphylococcus aureus y Staphylococcus. Epidermidis, donde se consideró solo el primer microorganismo aislado de cada paciente, y los porcentajes de resistencia para los antibióticos evaluados. Los puntos de corte para determinar la concentración mínima inhibitoria (CMI) de cada antibiótico, analizado mediante antibiograma por microdilución en caldo, fueron los recomendados por el Instituto de Estándares Clínicos y de Laboratorio (CLSI; del inglés, Clinical and Laboratory Standards Institute) versión 2014. Finalmente, se estableció el fenotipo de resistencia más prevalente observado en las bacterias objeto de estudio.
Este estudio fue aprobado por el comité de ética para investigación de la Universidad de Santander. Se consideró una investigación sin riesgo que no requería consentimiento informado. Las instituciones participantes firmaron la cláusula de confidencialidad.

\section{Resultados}

Se registraron un total de 3.245 muestras a las que les realizaron cultivos bacterianos durante el 2014, en los hospitales y clínicas adscritos a la Red de Laboratorios del Departamento del Cesar, 1.261 provenientes de la Unidad de Cuidados Intensivos y 1.984 de otros servicios. Los aislamientos bacterianos más frecuentes en las muestras provenientes de la Unidad de Cuidados Intensivos fueron Klebsiella pneumoniae (18,8\%), seguida de Pseudomonas aeruginosa $(15,0 \%)$ y Escherichia coli $(13,8 \%)$; mientras que en las muestras de los servicios diferentes a esta unidad se observó una mayor frecuencia de Escherichia coli $(36,4 \%)$, Staphylococcus aureus $(15,5 \%)$ y Klebsiella pneumoniae $(13,2 \%)$ (véase tabla 1$)$.

Los aislados de enterobacterias de KlebsieIla pneumoniae en la Unidad de Cuidados Intensivos presentaron una mayor resistencia a la ampicilina-sulbactam con $46,0 \%$, la cefepima y el aztreonam con $29,1 \%$ cada uno, y a las cefalosporinas de tercera generación (ceftazidima, ceftriaxona y cefotaxima) con valores de $28,3 \%$ para cada una de ellas; entretanto, aquellos provenientes de los servicios diferentes a la Unidad de Cuidados Intensivos presentaron resistencia para estos mismos antibióticos en el $43,1 \%$, $18,7 \%, 19,5 \%$ y $18,7 \%$, respectivamente. El fenotipo de resistencia de los aislamientos de Escherichia coli de la Unidad de Cuidados Intensivos para las cefalosporinas de terce- 


\begin{tabular}{|c|c|c|c|c|}
\hline \multirow[t]{2}{*}{ Microorganismos } & \multicolumn{2}{|c|}{$\begin{array}{l}\text { Unidad de Cuidados Intensivos } \\
(n=1.261)\end{array}$} & \multicolumn{2}{|c|}{$\begin{array}{l}\text { Servicios diferentes a la Unidad } \\
\text { de Cuidados Intensivos ( } n=1.984 \text { ) }\end{array}$} \\
\hline & N. ${ }^{\circ}$ & $\%$ & N. ${ }^{\circ}$ & $\%$ \\
\hline Klebsiella pneumoniae & 237 & 18,8 & 262 & 13,2 \\
\hline Pseudomonas aeruginosa & 189 & 15,0 & 221 & 11,1 \\
\hline Escherichia coli & 174 & 13,8 & 723 & 36,4 \\
\hline Staphylococcus aureus & 105 & 8,3 & 309 & 15,5 \\
\hline Acinetobacter spp. & 92 & 7,3 & 37 & 1,9 \\
\hline Serratia marcescens & 79 & 6,3 & 45 & 2,2 \\
\hline Staphylococcus epidermidis & 77 & 6,1 & 29 & 1,5 \\
\hline Burkholderia cepacia & 66 & 5,2 & 38 & 1,9 \\
\hline Stenotrophomonas maltophilia & 51 & 4,0 & 25 & 1,3 \\
\hline Enterobacter cloacae & 51 & 4,0 & 67 & 3,4 \\
\hline Enterococcus faecalis & 23 & 2,0 & 31 & 1,6 \\
\hline Proteus mirabilis & 22 & 1,7 & 67 & 3,4 \\
\hline Otros & 95 & 7,5 & 130 & 6,6 \\
\hline
\end{tabular}

ra (ceftazidima, ceftriaxona y cefotaxima) y cuarta generación (cefepima) fue del 21,8\% para cada una de ellas y para el aztreonam del $23,0 \%$, mientras que en los demás servicios la resistencia a estos antibióticos fue un poco menor, con valores alrededor del $17 \%$ para cada uno de ellos (véase tabla 2 ).

La mayor resistencia a los carbapenémicos de Klebsiella pneumoniae fue al meropenem, con un $3,4 \%$ de los aislamientos de la Unidad de Cuidados Intensivos, y al doripenem y al ertapenem, con un $1,5 \%$ cada uno en los demás servicios, en contraste con los aislados de Escherichia coli que no presentaron resistencia a estos antibióticos, excepto al doripenem en un $0,6 \%$ de los obtenidos en la Unidad de Cuidados Intensivos. La mayor resistencia a aminoglucósidos en estas dos enterobacterias en los dos servicios estudiados fue a la gentamicina, con 16,9\% y $19,0 \%$ en los aislados de Klebsiella pneumoniae y Escherichia coli de la Unidad de Cuidados Intensivos, y con $10,7 \%$ y $16,9 \%$, respectivamente, en los otros servicios. En cuanto a la ciprofloxacina la resistencia fue relativamente alta en los aislados de estas dos bacterias en los dos servicios de estudio, donde alcanzaron valores de resistencia del 30,5\% en los aislados de Escherichia coli obtenidos en la Unidad de Cuidados Intensivos (véase tabla 2).

En cuanto a los bacilos Gram negativos no fermentadores el género Acinetobacter mostró un mayor patrón de resistencia antimicrobiana que Pseudomonas aeruginosa, con un predominio de la resistencia a la ceftriaxona y la cefepima en el $51,1 \%$ de los aislados de Acinetobacter spp., obtenidos en la Unidad de Cuidados Intensivos, para cada cefalosporina. Frente al meropenem los aislados de Acinetobacter spp. presentaron una resistencia equivalente en los dos servicios, alcanzando un valor máximo de $46,7 \%$ en la Unidad de Cuidados Intensivos frente a un $43,2 \%$ en el resto de los servicios. En los aislados de Pseudomonas aeru- 


\begin{tabular}{|c|c|c|c|c|c|c|c|c|}
\hline \multirow[t]{3}{*}{ Antibióticos } & \multicolumn{4}{|c|}{ Klebsiella pneumoniae } & \multicolumn{4}{|c|}{ Escherichia coli } \\
\hline & \multicolumn{2}{|c|}{$\begin{array}{l}\text { Unidad de } \\
\text { Cuidados } \\
\text { Intensivos } \\
(n=237)\end{array}$} & \multicolumn{2}{|c|}{$\begin{array}{l}\text { Servicios diferentes a } \\
\text { la Unidad de Cuidados } \\
\text { Intensivos ( } n=262 \text { ) }\end{array}$} & \multicolumn{2}{|c|}{$\begin{array}{l}\text { Unidad de } \\
\text { Cuidados } \\
\text { Intensivos } \\
(\mathbf{n}=174)\end{array}$} & \multicolumn{2}{|c|}{$\begin{array}{l}\text { Servicios diferentes a } \\
\text { la Unidad de Cuidados } \\
\text { Intensivos ( } n=723 \text { ) }\end{array}$} \\
\hline & N. ${ }^{\circ}$ & $\%$ & N. ${ }^{\circ}$ & $\%$ & N. ${ }^{\circ}$ & $\%$ & N. ${ }^{\circ}$ & $\%$ \\
\hline Ampicilina-sulbactam & 109 & 46,0 & 113 & 43,1 & 70 & 40,2 & 291 & 40,2 \\
\hline Ceftazidima & 67 & 28,3 & 49 & 18,7 & 38 & 21,8 & 123 & 17,0 \\
\hline Ceftriaxona & 67 & 28,3 & 49 & 18,7 & 38 & 21,8 & 123 & 17,0 \\
\hline Cefotaxima & 67 & 28,3 & 49 & 18,7 & 38 & 21,8 & 123 & 17,0 \\
\hline Cefepima & 69 & 29,1 & 49 & 18,7 & 38 & 21,8 & 125 & 17,3 \\
\hline Piperacilina-tazobactam & 42 & 17,7 & 31 & 11,8 & 10 & 5,7 & 34 & 4,7 \\
\hline Aztreonam & 69 & 29,1 & 51 & 19,5 & 40 & 23,0 & 125 & 17,3 \\
\hline Doripenem & 7 & 3,0 & 4 & 1,5 & 1 & 0,6 & 0 & 0,0 \\
\hline Ertapenem & 6 & 2,5 & 4 & 1,5 & 0 & 0,0 & 0 & 0,0 \\
\hline Meropenem & 8 & 3,4 & 2 & 0,8 & 0 & 0,0 & 0 & 0,0 \\
\hline Amikacina & 2 & 0,8 & 22 & 8,4 & 4 & 2,3 & 7 & 1,0 \\
\hline Gentamicina & 40 & 16,9 & 28 & 10,7 & 33 & 19,0 & 122 & 16,9 \\
\hline Ciprofloxacina & 49 & 20,7 & 71 & 27,1 & 53 & 30,5 & 188 & 26,0 \\
\hline
\end{tabular}

ginosa los fenotipos de resistencia con mayor prevalencia se observaron también en la Unidad de Cuidados Intensivos y fueron más significativos para la ceftazidima $(27,0 \%)$, la cefepima $(21,2 \%)$, el meropenem $(19,0 \%)$ y la gentamicina $(20,1 \%)$, como se observa en la tabla 3.

La resistencia de los aislamientos de Staphylococcus aureus y Staphylococcus epidermidis a la penicilina fue similar entre ellas y en ambos servicios, encontrándose entre el $97,1 \%$ y el $100 \%$. En cuanto a la oxacilina la resistencia fue mayor en los aislados de Staphylococcus epidermidis de los servicios diferentes a la Unidad de Cuidados Intensivos, con un $89,7 \%$, y en esta unidad con un $81,8 \%$. La resistencia a este antibiótico también fue alta en los aislados de Staphylococcus aureus obtenidos en la Unidad de Cuidados Intensivos, con un $61,0 \%$, y en los demás servicios del $48,7 \%$. Los fenotipos de resistencia a la eritromicina y la clindamicina de Staphylococcus aureus fueron equivalentes en los dos servicios, con una resistencia del $21,9 \%$ y $10,5 \%$ en la Unidad de Cuidados Intensivos y del $20,1 \%$ y $9,1 \%$ en los otros servicios, respectivamente. En los aislamientos de Staphylococcus epidermidis se observó una mayor resistencia a estos dos antibióticos en la Unidad de Cuidados Intensivos, con un $72,7 \%$ para clindamicina y $71,4 \%$ para eritromicina (véase tabla 4 ).

\section{Discusión}

En este trabajo se determinó la frecuencia de microorganismos aislados de hospitales y clínicas del departamento del Cesar, don- 
Tabla 3. Número y porcentaje de los fenotipos de resistencia de Acinetobacter spp. y Pseudomonas aeruginosa según tipo de servicio hospitalario 2014

\begin{tabular}{|c|c|c|c|c|c|c|c|c|}
\hline \multirow[t]{3}{*}{ Antibióticos } & \multicolumn{4}{|c|}{ Acinetobacter spp. } & \multicolumn{4}{|c|}{ Pseudomonas aeruginosa } \\
\hline & \multicolumn{2}{|c|}{$\begin{array}{l}\text { Unidad de Cui- } \\
\text { dados Intensivos } \\
(n=92)\end{array}$} & \multicolumn{2}{|c|}{$\begin{array}{l}\text { Servicios diferentes a } \\
\text { la Unidad de Cuidados } \\
\text { Intensivos }(n=37)\end{array}$} & \multicolumn{2}{|c|}{$\begin{array}{l}\text { Unidad de Cuidados } \\
\text { Intensivos }(n=189)\end{array}$} & \multicolumn{2}{|c|}{$\begin{array}{l}\text { Servicios diferentes a } \\
\text { la Unidad de Cuidados } \\
\text { Intensivos }(n=221)\end{array}$} \\
\hline & N. ${ }^{\circ}$ & $\%$ & N. ${ }^{\circ}$ & $\%$ & N. ${ }^{\circ}$ & $\%$ & N. ${ }^{\circ}$ & $\%$ \\
\hline $\begin{array}{l}\text { Ampicilina- } \\
\text { sulbactam }\end{array}$ & 43 & 46,7 & 12 & 32,4 & $*$ & $*$ & $*$ & $*$ \\
\hline Ceftazidima & 27 & 29,3 & 9 & 24,3 & 51 & 27,0 & 50 & 22,6 \\
\hline Ceftriaxona & 47 & 51,1 & 14 & 37,8 & $*$ & $*$ & $*$ & $*$ \\
\hline Cefotaxima & 44 & 47,8 & 14 & 37,8 & $*$ & * & * & $*$ \\
\hline Cefepima & 47 & 51,1 & 15 & 40,5 & 40 & 21,2 & 41 & 18,6 \\
\hline Doripenem & 0 & 0,0 & 0 & 0,0 & 0 & 0 & 0 & 0 \\
\hline Meropenem & 43 & 46,7 & 16 & 43,2 & 36 & 19,0 & 25 & 11,3 \\
\hline Amikacina & 40 & 43,5 & 13 & 35,1 & 29 & 15,3 & 30 & 13,6 \\
\hline Gentamicina & 43 & 46,7 & 15 & 40,5 & 38 & 20,1 & 31 & 14 \\
\hline Ciprofloxacina & 41 & 44,6 & 12 & 32,4 & 22 & 11,6 & 27 & 12,2 \\
\hline $\begin{array}{l}\text { Trimetoprima- } \\
\text { sulfametoxazol }\end{array}$ & 48 & 52,2 & 18 & 48,6 & $*$ & $*$ & $*$ & $*$ \\
\hline$*=$ No evaluado. & $b a$ & esenta & ten & ca a este $n$ & ame & & & \\
\hline
\end{tabular}

de los más frecuentes dentro del grupo de las enterobacterias fueron Klebsiella pneumoniae y Escherichia coli; entre los bacilos Gram negativos no fermentadores Pseudomonas aeruginosa y entre los cocos Gram positivos Staphylococcus aureus; resultados que coinciden con otros estudios realizados en Colombia y Brasil $[7,8]$. Diversas investigaciones han reportado que Klebsiella pneumoniae es aislada con mayor frecuencia que Escherichia coli en el ambiente hospitalario, incluyendo las superficies inanimadas. Esto se debe a la mayor capacidad de las especies de Klebsiella para sobrevivir sobre las superficies a partir de las cuales puede colonizar los pacientes, en mayor o menor frecuencia, dependiendo de la especie bacteriana y de sus diferencias biológicas intrínsecas [9-11]. El problema se agrava cuando estos microorganismos expresan genes de resistencia a múltiples antimicrobianos, lo que convierte al ambiente hospitalario en un reservorio de bacterias multirresistentes de difícil erradicación, aún bajo la aplicación de los procedimientos más prolijos de limpieza al interior del hospital [10].

Al analizar los fenotipos de resistencia de los aislamientos obtenidos de Klebsiella pneumoniae y Escherichia coli se pudo observar un mayor patrón de resistencia a las cefalosporinas de tercera y cuarta generación y al aztreonam, especialmente en las unidades críticas. Este fenotipo de resistencia permite inferir que probablemente algunos de estos aislados son productores de betalactamasas de espectro extendido (BLEE), ya que está plenamente documentado que las bacterias portadoras de estas 


\begin{tabular}{|c|c|c|c|c|c|c|c|c|}
\hline \multirow[t]{3}{*}{ Antibióticos } & \multicolumn{4}{|c|}{ Staphylococcus aureus } & \multicolumn{4}{|c|}{ Staphylococcus epidermidis } \\
\hline & \multicolumn{2}{|c|}{$\begin{array}{l}\text { Unidad de Cuidados } \\
\text { Intensivos }(n=105)\end{array}$} & \multicolumn{2}{|c|}{$\begin{array}{l}\text { Servicios diferentes a } \\
\text { la Unidad de Cuidados } \\
\text { Intensivos }(n=309)\end{array}$} & \multicolumn{2}{|c|}{$\begin{array}{l}\text { Unidad de Cuidados } \\
\text { Intensivos }(n=77)\end{array}$} & \multicolumn{2}{|c|}{$\begin{array}{l}\text { Servicios diferentes a } \\
\text { la Unidad de Cuidados } \\
\text { Intensivos }(n=29)\end{array}$} \\
\hline & N. ${ }^{\circ}$ & $\%$ & N. ${ }^{\circ}$ & $\%$ & N. ${ }^{\circ}$ & $\%$ & $\mathbf{N} .^{\circ}$ & $\%$ \\
\hline Penicilina & 102 & 97,1 & 304 & 98,4 & 75 & 97,4 & 29 & 100,0 \\
\hline Oxacilina & 64 & 61,0 & 150 & 48,7 & 63 & 81,8 & 26 & 89,7 \\
\hline Gentamicina & 10 & 9,5 & 12 & 3,9 & 42 & 54,5 & 15 & 51,7 \\
\hline Rifampicina & 5 & 4,8 & 4 & 1,3 & 14 & 18,2 & 4 & 13,8 \\
\hline $\begin{array}{l}\text { Ciprofloxa- } \\
\text { cina }\end{array}$ & 8 & 7,6 & 10 & 3,2 & 34 & 44,2 & 6 & 20,7 \\
\hline $\begin{array}{l}\text { Trimetopri- } \\
\text { ma-sulfame- } \\
\text { toxazol }\end{array}$ & 5 & 4,8 & 8 & 2,6 & 40 & 51,9 & 14 & 48,3 \\
\hline Clindamicina & 11 & 10,5 & 28 & 9,1 & 56 & 72,7 & 20 & 69,0 \\
\hline Daptomicina & 0 & 0,0 & 0 & 0,0 & 0 & 0,0 & 0 & 0,0 \\
\hline Eritromicina & 23 & 21,9 & 62 & 20,1 & 55 & 71,4 & 19 & 65,5 \\
\hline Linezolid & 2 & 1,9 & 3 & 1,0 & 1 & 1,3 & 0 & 0,0 \\
\hline Vancomicina & 0 & 0,0 & 0 & 0,0 & 0 & 0,0 & 0 & 0,0 \\
\hline Tetraciclina & 32 & 30,5 & 90 & 29,1 & 21 & 27,3 & 8 & 27,6 \\
\hline
\end{tabular}

enzimas presentan resistencia a la mayoría de los betalactámicos, incluyendo las cefalosporinas de amplio espectro y el aztreonam, y altas tasas de resistencia a antimicrobianos de otras familias. Esto representa un problema terapéutico importante tanto en el ámbito hospitalario como en el comunitario, pues se dificulta el tratamiento de numerosas infecciones debido al perfil de multirresistencia que expresan las bacterias productoras de betalactamasas de espectro extendido (BLEE) [12-14].

El porcentaje de resistencia a las cefalosporinas de tercera generación en los aislados de Klebsiella pneumoniae fue inferior al encontrado en otras investigaciones realizadas en Colombia $[7,15,16]$ y contrario a lo reportado por Hernández-Gómez y colaboradores (2014) [17], quienes encontraron una prevalencia menor a la de este estudio. En cuanto a la resistencia a los carbapenémicos fue menor en comparación con otros estudios $[8,10,18]$; por el contrario, la resistencia a los aminoglucósidos se observó en una alta proporción, en contraste con los resultados obtenidos por Martínez-Buitrago y colaboradores (2014) [7]. Los porcentajes de resistencia de Escherichia coli a las cefalosporinas de tercera generación fueron proporcionales a los reportados por Hernández-Gómez y colaboradores (2014) [17], pero con valores superiores a las encontradas en otros estudios para las cefalosporinas, el aztreonam y la ciprofloxacina $[15,18]$. 
Este patrón de multirresistencia en Klebsiella pneumoniae y Escherichia coli supone una dificultad terapéutica que explica su asociación en numerosos estudios con una mayor mortalidad, retraso en el inicio del tratamiento antibiótico apropiado, aumento de la estancia hospitalaria y de los costos económicos directos e indirectos para las instituciones, el paciente y su familia. Esto significa que la resistencia bacteriana es un evento de interés en salud pública, el cual requiere del máximo esfuerzo de todas las instituciones gubernamentales para garantizar su control $y$ de las instituciones investigativas para que generen conocimiento y herramientas para el control y erradicación [15,19-22].

Acinetobacter spp. se ha convertido en un patógeno muy importante por su capacidad de colonizar e infectar a los pacientes, persistir en el medio ambiente hospitalario y presentar multirresistencia o panrresistencia a los antimicrobianos [23]. A excepción del doripenem, Acinetobacter spp. presentó una resistencia moderadamente alta a todos los antibióticos betalactámicos y no betalactámicos evaluados, incluyendo el meropenem, lo que evidencia la magnitud del problema de la resistencia a los antibióticos en este género bacteriano al interior de las instituciones prestadoras de servicios de salud [24-26]. Esta situación reafirma la importancia que tiene la vigilancia epidemiológica de la resistencia bacteriana a los antibióticos, como una herramienta para conocer y clasificar los perfiles de sensibilidad de los aislamientos e identificar los posibles mecanismos de resistencia que estos expresan, de manera que sea viable la contención de la resistencia a los antibióticos de las especies bacterianas, especialmente de Acinetobacter baumannii aislado en pacientes hospitalizados [27].

Pseudomonas aeruginosa es un patógeno oportunista de gran relevancia clínica que se encuentra entre los cinco microorganismos más aislados en los hospitales de Latinoamérica, incluyendo Colombia $[15,28]$. Comúnmente presenta resistencia a varias clases de antibacterianos, lo que dificulta el tratamiento y se asocia a mayores tasas de mortalidad e incrementos en el costo de la atención hospitalaria [29,30]. En este trabajo se observó un patrón de multirresistencia antibiótica en esta bacteria, con resistencia a las cefalosporinas de tercera generación ceftazidima, cefepima y carbapenémicos en cifras similares a las reportadas en otras investigaciones $[7,15]$, pero muy inferiores a lo documentado por Hernández-Gómez y colaboradores (2014) [17]. La resistencia a la ciprofloxacina fue bastante inferior en comparación con otros estudios realizados en Colombia $[7,15,17]$. En relación con los aminoglucósidos la frecuencia encontrada fue proporcional a la reportada por Martínez-Buitrago y colaboradores (2014) [7], donde la amikacina fue el antibiótico que lideró la resistencia; no obstante, en el presente estudio predominó la resistencia a la gentamicina.

La infección sanguínea por Pseudomonas aeruginosa resulta en una mayor tasa de mortalidad en los pacientes que reciben una terapia antimicrobiana empírica inadecuada, es decir, en los que se administran agentes a los que los aislados son resistentes in vitro o en los que no se usan agentes con actividad contra la bacteria, respecto a aquellos que reciben un tratamiento inicial apropiado $(30,7 \%$ frente a $17,8 \%$ ) [31]. Esto, sumado a la presión selectiva ejercida sobre las bacterias debido 
al uso indiscriminado de antimicrobianos en los hospitales, favorece el aumento de las infecciones por microorganismos multirresistentes en pacientes susceptibles. Por esta razón, también es importante conocer las tasas de resistencia locales y regionales para que se realice una prescripción apropiada y personalizada de los antimicrobianos [32].

Staphylococcus aureus es un importante patógeno nosocomial y comunitario, debido a las múltiples enfermedades que ocasiona. La alta resistencia a la penicilina encontrada en los aislados de Staphylococcus spp. de este trabajo es coherente con lo referenciado por múltiples investigaciones, donde la resistencia fue superior al $90 \%$ [7,33-35]. Por su parte, la resistencia a la oxacilina en los aislados de Staphylococcus aureus y Staphylococcus epidermidis de esta investigación fue mayor a la encontrada en otros estudios [15,36-38]. Esta discrepancia puede ser debida, probablemente, a las variaciones en las tasas de resistencia antibiótica entre un país y otro, a una mayor presión selectiva sobre las bacterias por el uso de antimicrobianos de amplio espectro, la no implementación de programas estructurados de vigilancia epidemiológica y las deficiencias en los protocolos encaminados al control de la infección en todas las áreas hospitalarias, los cuales han demostrado que reducen hasta en un $30 \%$ la tasa de infecciones hospitalarias [39].

Los porcentajes de resistencia a macrólidos y lincosamidas observados en este trabajo, en los aislamientos de Staphylococcus aureus y Staphylococcus epidermidis, son equivalentes a los encontrados por otros autores $[35,40]$ y representan implicaciones clínicas importantes para el paciente infectado ya que, por ejemplo, la clindamicina es una de las primeras líneas de tratamiento para las infecciones por estafilococos resistentes a la meticilina por lo que ante la resistencia a esta es necesario recurrir al uso de antibióticos costosos para el tratamiento de las mismas. Aunado a esto, la no detección in vitro del fenotipo inducible de resistencia a macrólidos, lincosaminas y estreptogramina B (MLSBi) en los aislamientos de Staphylococcus aureus conduce a fracasos terapéuticos producto de la inducción de resistencia in vivo a la clindamicina durante el tratamiento con este antibiótico [41].

Entre las limitaciones de este estudio se encuentra que no siempre se usaron los mismos antibióticos en las instituciones participantes, no estaban unificados los tipos de muestras que fueron analizadas y el perfil de resistencia de Acinetobacter baumannii no se pudo establecer, ya que las instituciones participantes reportaban solamente el género y no la especie.

\section{Conclusiones}

La alta resistencia antimicrobiana a los antibióticos de uso institucional, encontrada en este estudio, para el tratamiento de bacterias Gram positivas y Gram negativas, demuestra la necesidad de reforzar la vigilancia epidemiológica a nivel local para que las instituciones puedan implementar políticas y medidas de control que logren disminuir la presión selectiva ejercida sobre las bacterias, por el uso indiscriminado de los antibióticos, principal causa de la resistencia bacteriana en los ambientes hospitalarios y la transmisión de infecciones causadas por bacterias multirresistentes. Por tal razón, realizar la confirmación 
molecular de los fenotipos de resistencia bacteriana permitirá fortalecer el Sistema Nacional de Vigilancia en Salud Pública. De esta manera, el laboratorio de microbiología desempeña un papel determinante para la identificación de los perfiles de resistencia bacterianos, los cuales van a brindar la información necesaria para darle al paciente un tratamiento acertado y oportuno, y limitar el uso inadecuado de antibióticos de amplio espectro, lo que a su vez impide que se favorezca el aumento de microrganismos resistentes a nivel hospitalario.

\section{Agradecimientos}

A la Universidad de Santander, al Laboratorio de Salud Pública del Departamento del Cesar y a la Red de Laboratorios Públicos y Privados, quienes hicieron posible esta investigación.

\section{Bibliografía}

1. Organización Mundial de la Salud. Estrategia mundial de la OMS para contener la resistencia a los antimicrobianos. 2001. Disponible: http:// www.antibioticos.msc.es/PDF/resist_OMS_estrategia mundial_resumen.pdf. Consultado: agosto 2017.

2. World Health Organization. Antimicrobial Resistance: Global Report on Surveillance. 2014. Disponible: http://apps.who.int/iris/bitstre am/10665/112642/1/9789241564748_eng.pdf. Consultado: agosto 2017.

3. McGowan JE, Jr., Carlet J. Antimicrobial resistance: a worldwide problem for health care institutions. Am J Infect Control 1998; 26: 541-543.

4. Villalobos AP, Barrero LI, Rivera SM, Ovalle MV, Valera D. Vigilancia de infecciones asociadas a la atención en salud, resistencia bacteriana y consumo de antibióticos en hospitales de alta complejidad, Colombia, 2011. Biomed 2014; 34: 67-80.
5. Richards M, Thursky K, Buising K. Epidemiology, prevalence, and sites of infections in intensive care units. Semin Respir Crit Care Med 2003; 24: 3-22.

6. Bearman GM, Munro C, Sessler CN, Wenzel RP. Infection control and the prevention of nosocomial infections in the intensive care unit. Semin Respir Crit Care Med 2006; 27: 310-324.

7. Martínez-Buitrago E, Hernández C, Pallares C, Pacheco R, Hurtado K, Recalde M. Frecuencia de aislamientos microbiológicos y perfil de resistencia bacteriana en 13 clínicas y hospitales de alta complejidad en Santiago de Cali - Colombia. Infect 2014; 18: 3-11.

8. Lopes-Campos e Vieira HP, Bezerra Magalhães J. Avaliação da resistência microbiana em hospitais privados de Fortaleza - Ceará. Rev Bras Farm 2013; 94: 83-87.

9. Freeman JT, Nimmo J, Gregory E, Tiong A, De Almeida M, McAuliffe GN, et al. Predictors of hospital surface contamination with Extended-spectrum beta-lactamase-producing Escherichia coli and Klebsiella pneumoniae: patient and organism factors. Antimicrob Resist Infect Control 2014; 3: 5.

10. López-Cerero L. Papel del ambiente hospitalario y los equipamientos en la transmisión de las infecciones nosocomiales. Enferm Infecc Microbiol Clin 2014; 32: 459-464.

11. Rivera-Jacinto MA. [Extended-spectrum beta-lactamases in isolates of Escherichia coli and Klebsiella sp. from inanimate reservoir at a hospital in northern Peru]. Rev Esp Quimioter 2012; 25: 161-163.

12. Morejón-García M. Betalactamasas de espectro extendido. Rev Cubana Med 2013; 52: 272-280.

13. Amado NY, Fajardo HD, Ramírez R RY, González P GI. Prevalencia de betalactamasas de espectro extendido en bacilos gramnegativos de una institución de salud de Tunja (Colombia) en el año 2013. Salud Soc Uptc 2014; 1: 54-60.

14. Molina L, Barragan I, Sánchez M. Comportamiento epidemiológico y clínico de las infecciones por E. Coli productora de betalactamasa de espectro extendido (BLEE) en la Clínica Universitaria San Juan de Dios de enero 2010 a diciembre de 2011. Espiga Científica 2014; 9 (2).

15. Villalobos-Rodríguez AP, Díaz-Ortega $\mathbf{M H}, \mathbf{B a}$ rrero-Garzón LI, Rivera-Vargas SM, Henríquez- 
Iguarán DE, Villegas-Botero MV, et al. Tendencias de los fenotipos de resistencia bacteriana en hospitales públicos y privados de alta complejidad de Colombia. Rev Panam Salud Publica 2011; 30: 627-633.

16. González L, Cortés JA. Revisión sistemática de la farmacorresistencia en enterobacterias de aislamientos hospitalarios en Colombia. Biomed 2014; 34: 180-197.

17. Hernández-Gómez C, Blanco VM, Motoa G, Correa A, Maya JJ, de la Cadena E, et al. Evolución de la resistencia antimicrobiana de bacilos Gram negativos en unidades de cuidados intensivos en Colombia. Rev Biomed 2014; 34: 91-100.

18. World Health Organization. World Health Organization. Report on the burden of endemic health care-associated infection worldwide. 2014. Disponible: http://apps.who.int/iris/bitst ream/10665/80135/1/9789241501507_eng.pdf. Consultado: agosto 2017.

19. Rottier WC, Ammerlaan HS, Bonten MJ. Effects of confounders and intermediates on the association of bacteraemia caused by extended-spectrum beta-lactamase-producing Enterobacteriaceae and patient outcome: a meta-analysis. J Antimicrob Chemother 2012; 67: 1311-1320.

20. Schwaber MJ, Navon-Venezia S, Kaye KS, BenAmi R, Schwartz D, Carmeli Y. Clinical and economic impact of bacteremia with extended-spectrum-beta-lactamase-producing Enterobacteriaceae. Antimicrob Agents Chemother 2006; 50: 1257-1262.

21. Perianes-Díaz ME, Novo-Veleiro I, Solís-Díaz K, Prolo-Acosta A, García-García I, Alonso-Claudio G. Bacteriemia por Escherichia coli y Klebsiella pneumoniae productoras de betalactamasas de espectro extendido: factores asociados a mortalidad y reingreso hospitalario. Med Clin 2014; 142: 381-386.

22. Garcia-Castellanos T, Castillo-Marshal A, Salazar-Rodríguez D. Mecanismos de resistencia a betalactámicos en bacterias gramnegativas. Rev Cubana Salud Pública 2014; 40: 129-135.

23. Ramírez-Sandoval MLP, Aranza-Aguilar J, Varela-Ramirez M. Brote de infección nosocomial de vías respiratorias bajas por Acinetobacter baumannii en un servicio de Medicina Interna de un hospital general de la Ciudad de México. Med Int Méx 2013; 29: 250-256.
24. Higgins PG, Dammhayn C, Hackel M, Seifert H. Global spread of carbapenem-resistant Acinetobacter baumannii. J Antimicrob Chemother 2010; 65: 233-238.

25. Organización Panamericana de la Salud. Informe Anual de la Red de Monitoreo/Vigilancia de la Resistencia a los Antibióticos. 2009. Disponible: http://www.paho.org/hq/ index.php?option=com_docman\&task $=$ doc view\&gid=14877\&Itemid=4108. Consultado: ago 2017.

26. Chávez M, Salazar MC. Bacterias resistentes a los antibióticos en infecciones nosocomiales de un hospital en Colombia. Enf Inf Microbiol 2013; 33: 19-25.

27. Prado A, Arias NL, Chávez M, Cabrera CE, Gómez RF. Caracterización fenotípica de aislamientos de Acinetobacter baumannii en una institución de salud de alta complejidad de Cali. Biomed 2014; 34: 101-107.

28. Gales AC, Castanheira M, Jones RN, Sader HS. Antimicrobial resistance among Gram-negative bacilli isolated from Latin America: results from SENTRY Antimicrobial Surveillance Program (Latin America, 2008-2010). Diagn Microbiol Infect Dis 2012; 73: 354-360.

29. Riou M, Carbonnelle S, Avrain L, Mesaros N, Pirnay JP, Bilocq F, et al. In vivo development of antimicrobial resistance in Pseudomonas aeruginosa strains isolated from the lower respiratory tract of Intensive Care Unit patients with nosocomial pneumonia and receiving antipseudomonal therapy. Int J Antimicrob Agents 2010; 36: 513-522.

30. Cortes JA, Cuervo SI, Urdaneta AM, Potdevin G, Arroyo $P$, Bermudez D, et al. Identifying and controlling a multiresistant Pseudomonas aeruginosa outbreak in a Latin-American cancer centre and its associated risk factors. Braz J Infect Dis 2009; 13: 99-103.

31. Micek ST, Lloyd AE, Ritchie DJ, Reichley RM, Fraser VJ, Kollef $\mathbf{M H}$. Pseudomonas aeruginosa bloodstream infection: importance of appropriate initial antimicrobial treatment. Antimicrob Agents Chemother 2005; 49: 1306-1311.

32. Villa LM, Cortés JA, Leal AL, Meneses A, Meléndez MP. Pseudomonas aeruginosa resistente a antimicrobianos en hospitales colombianos. Rev Chil Infectol 2013; 30: 605-610. 
33. Casellas JM. Resistencia a los antibacterianos en América Latina: consecuencias para la infectología. Rev Panam Salud Publica 2011; 30: 519-528.

34. Chambers HF, DeLeo FR. Waves of Resistance: Staphylococcus aureus in the Antibiotic Era. Nat Rev Microbiol 2009; 7: 629-641.

35. Fariña N, Carpinelli L, Samudio M, Guillén R, Laspina F, Sanabria R, et al. Staphylococcus coagulasa-negativa clínicamente significativos: Especies más frecuentes y factores de virulencia. Rev Chil Infectol 2013; 30: 480-488.

36. Grupo para el Control de la Resistencia Antibacteriana en Bogotá. Información de resistencia bacteriana GREBO año 2015. 2015. Disponible: http://www.grebo.org/grebo_site/jgrebo/documentos/Boletin_Grebo_2015.pdf. Consultado: agosto 2017.

37. Grupo para el Control de la Resistencia Antibacteriana en Bogotá. Información de resistencia bacteriana GREBO año 2014. 2014. Disponible:
http://www.grebo.org/documentos/Boletin_ Grebo_2014.pdf. Consultado: agosto 2017.

38. República de Colombia, Alcaldía Mayor de Bogotá D.C., Secretaría Distrital de Salud. Boletín epidemiológico de Resistencia Bacteriana (CIVIBAC) 2012- 2013. 2014. Disponible: http://www. saludcapital.gov.co/DSP/Resistencia\%20Bacteriana/Boletines/Bolet\%C3\%ADn\%20IAAS\%20 2014.pdf. Consultado: agosto 2017.

39. Pujol M, Limón E. Epidemiología general de las infecciones nosocomiales. Sistemas y programas de vigilancia. Enferm Infecc Microbiol Clin 2013; 31: 108-113.

40. Arslan S, Ozkardes F. Slime production and antibiotic susceptibility in staphylococci isolated from clinical samples. Mem Inst Oswaldo Cruz 2007; 102: 29-33.

41. Morosini MI, Cercenado E, Ardanuy C, Torres C. Detección fenotípica de mecanismos de resistencia en microorganismos grampositivos. Enferm Infecc Microbiol Clin 2012; 30: 325-332.

Introduction: Antimicrobial resistance is a public health problem that implies difficult and expensive treatments. Objective: to analyze the antimicrobial resistance phenotypes in bacterias isolated in hospitals and clinics in the department of Cesar (Colombia) during 2014. Materials and methods: $A$ descriptive study was conducted in five clinical laboratories of the department. Data were analyzed using Whonet 5.6 software and profiles of bacterial resistance were reported. Results: Gram-negative bacteria more isolated in Intensive Care Unit were Klebsiella pneumoniae (18.8\%), Pseudomonas aeruginosa (15.0\%) and Escherichia coli (13.8\%). In the non-Intensive Care Unit service Escherichia coli (36.4\%) and Staphylococcus aureus (15.5\%) predominated. In the Intensive Care Unit service a higher resistance was obtained to ampicillin-sulbactam and third and fourth generation of cephalosporins in Klebsiella pneumonia isolates (28.3\%, 29.1\% and 29.1\%, respectively), and Escherichia coli isolates (21.8\%, 21.8\% y 23.0\%, respectively). In Acinetobacter isolates the resistance was higher than Pseudomonas aeruginosa isolates, with a predominance of resistance to ceftriaxone and cefepime (51.1\%) of Intensive Care Unit isolates. In Staphylococcus aureus and Staphylococcus epidermidis isolates the resistance to oxacillin were observed in $61.0 \%$ y $81.8 \%$ in Intensive Care Unit isolates and $48.7 \%$ and $89.7 \%$ in the others services, respectively. Conclusions: High frequencies of antibiotic resistance were observed. Therefore, reinforcing local epidemiological surveillance will allow decreasing bacterial resistance in hospital settings.

Key words: bacterial drug resistance, epidemiological surveillance, Gram-positive bacteria, Gramnegative bacteria. 